\title{
Proposing a Fuzzy Adaptation Mechanism Based on Cognitive Factors of Users for Web Personalization
}

\author{
Efi Papatheocharous, Marios Belk, Panagiotis Germanakos, and George Samaras \\ Department of Computer Science, University of Cyprus, \\ P.O. Box 20537, 1678 Nicosia, Cyprus \\ \{efi.papatheocharous, belk, pgerman, cssamara\} @cs.ucy.ac.cy
}

\begin{abstract}
The increased demand of Web services and diverse characteristics of users have resulted in a plethora of applications that aim to provide personalized services based on the heterogeneous needs and preferences of users. With the aim to enhance and support the personalization process of Web applications, an innovative adaptation mechanism is proposed. The mechanism is based on a series of psychometric measures which capture the cognitive style of users and a Computational Intelligence technique embracing Artificial Neural Networks and Fuzzy Logic. The proposed mechanism decides on the adaptation effects of Web applications and provides a personalized user experience. The proposed method has been evaluated with a user study and provides interesting insights with respect to the effect of adaptation in terms of task accuracy, performance and satisfaction of users while interacting with an adapted and a non-adapted version of the same Web environment.
\end{abstract}

Keywords: User Modeling, Adaptation, Personalization, Cognitive Styles, Artificial Neural Networks, Fuzzy Logic.

\section{Introduction}

User-adaptive Web systems attract many researchers from different communities since the mid-1990s due to the exponential increase of content and usage of the World Wide Web [1]. In this context, researchers and practitioners from different communities, like hypermedia, user modeling, machine learning, information retrieval, cognitive science, and Web-based education, aim to provide an alternative to the "one size fits all" approach of static user interfaces by adapting the system's content and functionality based on the users' needs and preferences.

The high-level research goals of User-adaptive systems are focused around two main issues; appropriate user modeling dealing with what information is important to be incorporated in the user model and how it can be represented and extracted, and appropriate adaptation procedures dealing with what adaptation types and mechanisms are most effective to be performed and how they can be translated into adaptive user interface designs in order to improve the system's usability and provide a positive user experience. 
The work presented in this paper lies on previous research [2] that has shown that specific cognitive factors may have significant impact in the adaptation and personalization process of User-adaptive systems. Specifically, since content and functionality of Web applications is either presented visually or verbally, and users may have specific navigation behavior, e.g. linear or nonlinear navigation behavior, the work in [2] has suggested that cognitive styles of users, which describe the way individuals, perceive and organize information [3], might be applied effectively for designing adaptive Web applications. The main outcome from the work conducted has shown that the adapted version of a Web environment based on the users' cognitive styles has increased their satisfaction, task accuracy and performance.

Follow up work of the authors in [2] is to support the adaptation process with a Computational Intelligence technique embracing Artificial Neural Networks (ANNs) and Fuzzy Logic. As in [2], the overarching aim of this work is to study the effect of adapting content and functionality of Web applications based on cognitive styles of users. While the work in [2] utilized a deterministic rule-based adaptation mechanism to provide the adaptation effects based on cognitive characteristics of users, main aim of this work is to extend the adaptation mechanism and exploit the benefits of partial truth by employing the use of Fuzzy Logic in the adaptation process, and investigate whether it increases usability and user experience of Web applications.

The paper is organized as follows: In Section 2, we provide an overview of adaptation mechanisms. Section 3 presents the proposed adaptation mechanism which is evaluated with a user study in Section 4. Consequently, we conclude the paper and describe our directions of future work in Section 5.

\section{Adaptation Mechanisms}

Adaptation mechanisms apply specific algorithms that decide what adaptation will be performed on the content and functionality of the system. Various approaches have been proposed in the literature, including among others rule-based, content-based and collaborative mechanisms.

Rule-based mechanisms refer to the process of producing high-level information from a set of low-level metrics, related to both static and dynamic user context information. Bearing in mind that the dynamic part of the context data model can be updated in real time it becomes obvious that reasoning capabilities supported provide an added value supporting users in different tasks. Such rules can initiate automated system actions or compare predictive user interaction models with actual user interaction data gathered in real time, providing thus valuable insights related to the current user goals and efficiency of interactions. For example, an online banking system may contain a rule as follows: "IF [USER].logged = False AND [USER].loginattempts.count > 2 THEN [UIOBJECT].LiveSupport.show = True", which indicates that the system should automatically offer a live customer support option to users who could not succeed to login in the system after trying to login for more than two times. A detailed analysis and comparison of rule-based mechanisms can be found in [4]. 
Content-based mechanisms suggest labeling of links by analyzing the content of Web-pages. A typical content-based mechanism includes the following steps: i) prefetch the content behind the links of the current page, ii) parse the pre-fetched pages to create a weighted keyword vector of each page, iii) compare the weighted keyword vector of each page with the user's preferences, that are also usually represented using a weighted keyword vector, and iv) suggest pages whose keyword vectors are the same with the user's preferences. A detailed analysis and comparison of contentbased mechanisms can be found in [5].

Collaborative mechanisms exploit the social process of people recommending something they have been experienced with (e.g., read a book, watched a movie, etc.) to other people. Collaborative mechanisms are based on the assumption that if users $X$ and $Y$ rate $n$ items similarly, or have similar behaviors (e.g., buying, watching), they will have similar interests. A survey of collaborative mechanisms can be found in [6].

To this end, this work suggests utilization of Computational Intelligence techniques initially to cluster users of similar type in specific categories and then to propose a method to avoid the use of deterministic truth in such adaptation rules mentioned in the rule-based adaptation approach. Accordingly, we propose and employ the use of Fuzzy Logic [7] to support the adaptation process of Web applications and exploit the benefits of partial truth. The advantage of the technique is that users are clustered to belong in more than one cluster (using fuzzy memberships) and the reasoning of the adaptation follows a fuzzy approach which is similar to the real decision making process humans follow.

\section{Proposed Fuzzy Adaptation Mechanism}

In this section we describe a novel fuzzy adaptation mechanism (Figure 1) with the aim to provide adapted content and functionality of Web applications based on user models which include cognitive factors.

The mechanism includes two main components; i) user modeling, and ii) fuzzy system for supporting the adaptation rules which define the adaptation effects.

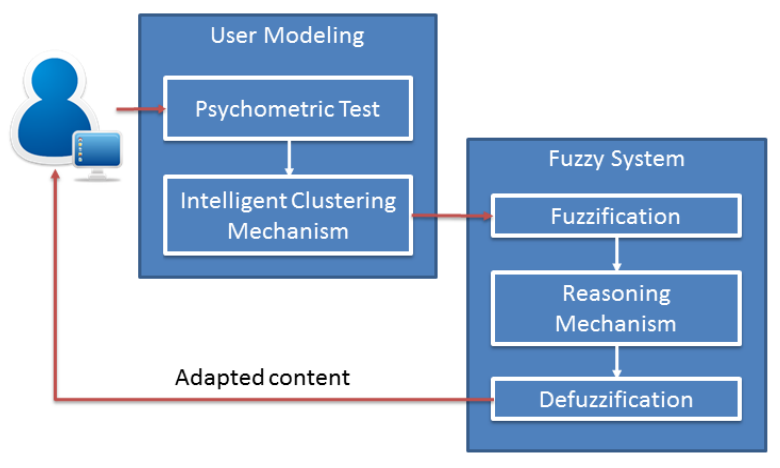

Fig. 1. Fuzzy Adaptation Mechanism 


\subsection{User Modeling}

For user modeling a series of psychometric tests are used to highlight differences in the cognitive characteristics of users. Among the popular theories of individual styles proposed [3][8][9][10], the current work utilizes Riding's Cognitive Style Analysis (CSA) and Baddeley's Working Memory Span (WMS) [10] which are discussed next.

Cognitive Styles. Riding's CSA consists of two dimensions; the Verbal/Imager dimension refers to how individuals process information, and the Wholist/Analyst dimension refers to how individuals organize information [3]. The Verbal/Imager dimension consists of three classes, users that belong to the Verbal, Intermediate or Imager class. Users that belong to the Verbal class can proportionally process textual and/or auditory content more efficiently than images, whereas users that belong to the Imager class the opposite. Users that belong in between the two end points (i.e., Intermediate) do not differ significantly with regards to information processing. The Wholist/Analyst dimension consists of three classes, users that belong to the Wholist, Intermediate or Analyst class. Specifically, users that belong to the Wholist class view a situation and organize information as a whole and are supposed to take a linear approach in hypermedia navigation. Users that belong to the Analyst class view a situation as a collection of parts, stress one or two aspects at a time and are supposed to take a nonlinear approach in hypermedia navigation. Users that belong in between the two end points of the Wholist/Analyst scale (i.e., Intermediate) do not differ significantly with regards to information organization.

In this regards, we consider that Riding's CSA [3] implications can be mapped on Web environments, since they consist of distinct scales that respond directly to different aspects of the Web space. The CSA implications can provide clear guidelines in the context of Web design (i.e., selecting to present visual/verbal content and structuring information flow in a wholistic/analytic manner), and is probably one of the most inclusive theories, since it is actually derived from the common axis of a number of theories [11].

Working Memory Span. "The term working memory refers to a brain system that provides temporary storage and manipulation of the information necessary for such complex cognitive tasks as language comprehension, learning, and reasoning" [12]; Baddeley also refers to individual differences in the Working Memory Span (WMS) of the population, thus providing a very good argument for using this concept as a personalization factor. Each corresponding WMS instance (i.e., low/medium/high), indicates the working memory capacity of a person (e.g., a person with low WMS has low working memory capacity and so on). In addition, WMS has implications on the navigation, quantity of content and aesthetics of a Web application.

Intelligent Clustering. Artificial Neural Networks (ANNs) [13] are primarily used to classify users in specific categories of cognitive style (e.g., assign users to categories of the Verbal/Imager dimension). The mechanism accordingly calculates the membership degree of each user in each group of cognitive typology. Thus, users belong at the same time to more than one group but with respect to a specific degree (introducing the notion of fuzziness). In contrast to classification, i.e., in hard clustering, where users are divided 
into distinct groups, in fuzzy clustering (or soft clustering) users belong to more than one group [14]. The users' respective membership degree or level informs us on the strength of the association between the users and categories representing the cognitive typology dimension.

In particular, the ANNs developed are pattern recognition feedforward networks [13] with one input, one hidden and one output layer. The rest parameters of the ANNs developed are: the number of neurons in the hidden layer varied from 2-20 neurons, the transfer function was the hyperbolic tangent sigmoid (tansig), the training function is the Levenberg-Marquardt backpropagation (trainlm), the number of epochs is set to 500, the backpropagation weight/bias learning function is the Gradient descent with momentum backpropagation (learngdm) and the performance function of the network is based on the weight sum of two factors: the mean squared error and the mean squared weight and bias values (msereg).

\subsection{Fuzzy System}

Several options exist for developing a reasoning mechanism, e.g., probabilistic, Bayesian reasoning and fuzzy logic modeling. Accordingly, in this work the preferred method for adapting the environment of Web applications for individuals is Fuzzy Logic because human behaviour is usually characterized in fuzzy terms (i.e., Person A is more Verbal than Person B and Person C is a lot more Verbal than Person B would be used to describe the Verbal/Imager dimension of three individuals from the scale low, medium, high in the following order: B, A and C). Accordingly, a person is not necessarily part of a Verbal or an Imager class, but we feel that a person is better described as belonging to a Verbal class in a certain degree (i.e., 60\%) and to an Imager class in another degree (e.g., 40\%). To better explain this, we illustrate an example of how the decision or reasoning mechanism works on the one hand through crisp and on the other hand by fuzzy logic.

Suppose that an individual has a cognitive style ratio equal to 1.09. In crisp logic and for the Verbal/Imager dimension, this individual is considered to be an Imager. Figure 2 shows (a) how an individual is classified in a crisp class (left part) and (b) how an individual is characterized as low-Imager and belongs in a fuzzy class (right part).
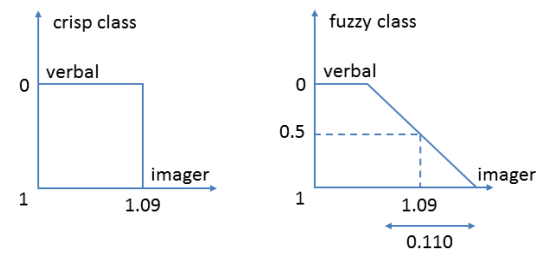

Fig. 2. Example of a Crisp and a Fuzzy Class for the Verbal/Imager Dimension

Accordingly, in this work the predicted values of membership degrees to classes yield from the ANNs are represented by memberships in fuzzy logic classes. A simple approach is followed for applying fuzzy logic principles; we consider only three or five values for the number of membership functions for each dimension (i.e., low, medium, 
high and very low, low, medium, high and very high). Then, this percentage of participation is used for adapting the environment of Web applications in small, medium, or high degree according to the membership value obtained. In the crisp case, the decision mechanism would include rules of the form: "If an individual is Verbal then provide all content in a textual form" while in the fuzzy case the rule would be "If an individual is low-Verbal and high-Imager then provide all content through diagrams and images" which is definitely more informative and useful. In addition, the reasoning mechanism, provided by a group of experts, includes a set of rules taking into consideration: a) the Verbal/Imager dimension, b) the Analyst/Wholist dimension, and c) the Working Memory Span (WMS).

The fuzzy approach is applied in calculating the relative membership of each user in the three categories of cognitive typologies (i.e., Verbal, Intermediate and Imager, and Wholist, Intermediate and Analyst) and three categories of Working Memory Span (i.e., Low, Medium and High). The approach introduces linguistic values stimulated by the membership functions as explained above and as shown in Table 1 and Figures 3-5.

Table 1. Values of the Bounds of the Membership Functions

\begin{tabular}{llllllllllll}
\hline & $\mathrm{a} 1$ & $\mathrm{~b} 1$ & $\mathrm{c} 1$ & $\mathrm{~d} 1$ & $\mathrm{a} 2$ & $\mathrm{~b} 2$ & $\mathrm{c} 2$ & $\mathrm{a} 3$ & $\mathrm{~b} 3$ & $\mathrm{c} 3$ & $\mathrm{~d} 3$ \\
\hline Wholist/Analyst & -2.67 & -2.27 & 2.01 & 1.02 & 1.02 & 1.18 & 1.35 & 1.25 & 1.35 & 2.01 & 2.01 \\
Verba1/Imager & -2.67 & -2.27 & 0.98 & 0.99 & 0.95 & 1.03 & 1.09 & 1.05 & 1.09 & 2.08 & 2.72 \\
WMS & -2.67 & -2.27 & 5 & 9 & 5 & 10.5 & 16 & 14 & 16.14 & 22 & 23 \\
\hline
\end{tabular}

The process followed for fuzzification is based on triangular and trapezoidal membership functions, as described in equations (1) and (2) respectively:

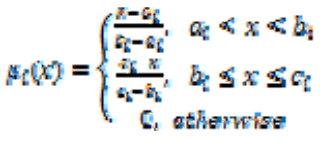

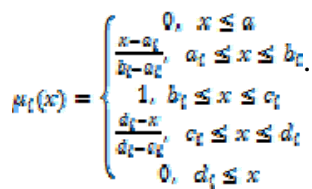

In triangular memberships the function is estimated using three values, $a, b$ and $c$ which represent the left, centre and right points of the function.

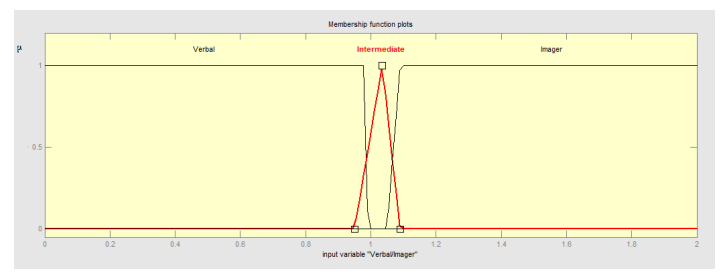

Fig. 3. Trapezoidal and Triangular Membership Functions Used for the Verbal/Imager Types 
In trapezoidal memberships the function is estimated using four values, $a, b, c$ and $d$ which represent the left, the left shoulder, the right shoulder and the right points of the function. The shape was chosen empirically by experts who also defined the bounds of the membership functions (Table 1).

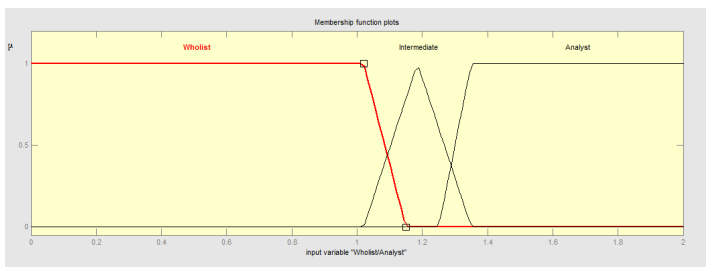

Fig. 4. Trapezoidal and Triangular Membership Functions Used for the Wholist/Analyst Types

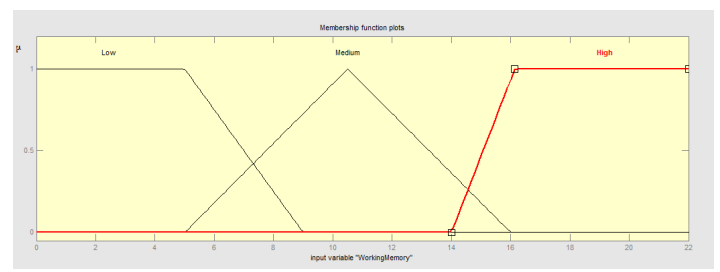

Fig. 5. Trapezoidal and Triangular Membership Functions Used for the Working Memory Span

The process of defuzzification translates back the membership values of the corresponding fuzzy sets into a specific adaptation decision. In particular, based on the theory of individual styles described in the previous section, this work suggests the adaptation effects, illustrated in Table 2, which compose the reasoning mechanism using a set of fuzzy rules.

Table 2. Design Implications based on Cognitive Styles Theory

\begin{tabular}{llllll}
\hline Imager & Verbal & Analyst & Wholist & Intermediate & WMS (low) \\
\hline Presentation of & Usage of & Content & Content & Receive a & Receive a \\
information is & text, & structure is & structure & condition & tool for \\
visually & without & chunked to & follows a & balanced & adding \\
enhanced in a & visual & clear-cut links & more & between the & hyperlinks \\
diagrammatical & enrichment & to match & holistic & opposite & of the \\
form of & & analytical way & pattern & preferences & section user \\
representation & & of thinking & & & is visiting \\
\hline
\end{tabular}

Accordingly, an example rule is: "IF a user is high-Imager AND has low WMS THEN provide him/her an additional tool that allows him/her to make entries of goalrelated information in a diagrammatical representation". Accordingly, this approach enables users with low WMS to organize and access large amounts of information, alleviating disorientation and cognitive load caused by the high amount of information. The process utilized the AND operator for calculating the minimum 
value of the partial membership functions of the user characteristics, while the maximum function was used for the corresponding OR parts of the rules for deciding on the adaptation effects.

To this end, the mechanism proposed is used for deciding on the adaptation effects of Web applications using notions of Fuzzy Logic. It reproduces human decision reasoning and includes concepts of partial truth. The main benefit is that it may continuously adapt the environment using new data as they become available and it is able to represent different levels of uncertainty while using the same decision model (rules and membership functions) and may thus cater for the particular needs of precision in different contexts and environments.

\section{$4 \quad$ User Study}

The aforementioned adaptation mechanism has been evaluated through a user study to investigate the effect of adaptation in Web applications based on human factors.

\subsection{Method of Study}

The study was carried out during the month of March 2012. The sample included 29 Computer Science students of age 22-25. The participation was voluntarily. All users accessed a Web-site utilized for the study with personal computers located at the laboratories of the University. The students first provided their demographic characteristics (i.e., name, age, education, etc.) and performed a number of interactive tests using attention and cognitive processing psychometric tools in order to quantify their cognitive characteristics described in the previous section. Based on the responses of the users, an ANN was trained and yielded the participation degree of each user in each cognitive style group with regards to the Verbal/Imager, Analyst/Wholist, and Working Memory Span (WMS) dimension. Finally, the adaptation was decided through the fuzzy rules and then, the students were asked to navigate in a reproduced replica of a commercial Web-site selling computer products. The Web-site's content concerned a series of laptop computers and included information such as general description and technical specifications.

The students navigated in two different versions of the same environment (i.e., original or personalized) and were asked to fulfill three tasks in each version. In particular, they had to find the necessary information to answer three sequential multiple choice questions that were given to them while navigating. All six questions were about determining which laptop excelled with respect to the prerequisites that were set by each question. The selection process of the sequence of version per individual was based on a random selection process. As soon as users finished answering all questions in both versions, they were presented with a comparative satisfaction questionnaire based on WAMMI questionnaire [15]; users were asked to choose which environment was better (using a scale from 1-5, where 1 means strong preference for environment A - original and 5 for environment B - personalized), regarding usability factors. The dependent variables utilized as indicators of differences between the two versions were: i) Task accuracy, ii) Task completion time. 


\subsection{Results}

In order to assess the significance and possible impact cognitive factors may have on the adaptation of content and functionality of Web applications in terms of task accuracy, a comparison has been performed between the average correct answers the users provided in each version (i.e., original and personalized). Users in the personalized version were more accurate in providing the correct answer for each task. In particular, users in the original version had a mean of 2.48 correct answers (out of 3), while in the personalized version the mean rose to 2.66. A further analysis was conducted that aimed to compare the average correct answers per user group in each version. The analysis revealed that all user groups had more correct answers in the personalized version except users that had low working memory (WMS).

Although the difference of accuracy between the two versions is not significant, this is a rather encouraging finding for the proposed mechanism, implying that adaptation on the basis of these factors (cognitive style and WMS) provides adaptation effects that benefits users within an eCommerce environment. A further analysis with a greater sample is required in order to draw more concrete conclusions. Equally interesting is the fact that users in the personalized condition were slightly faster at task completion. The mean aggregated time of answering all three questions was 66.3 seconds in the original condition, and 66 in the personalized. Again, this dependent variable (time) shows that the proposed mechanism provides accurate adaptation effects based on the cognitive characteristics of users.

Finally, a questionnaire was utilized to retrieve the users' perceptions regarding the two environments (i.e., original vs. adapted). In particular, users were asked in which environment they could find easier and faster the information they were looking for. Accordingly, based on the results, $69 \%$ of the users stated that the personalized version helped them to find the information they were looking for, $24 \%$ claimed the original version was easier, whereas $7 \%$ didn't observe any difference between the two versions. Furthermore, results also revealed that 59\% of the users could find the information they were looking faster in the personalized version, $31 \%$ in the original version and $10 \%$ did not observe any difference between the two versions.

\section{Conclusions}

The basic objective of this paper was to propose a Computational Intelligence technique which utilizes Artificial Neural Networks and Fuzzy Logic for developing and adapting Web applications based on a combination of human factors, namely cognitive styles and working memory. A user study utilizing the proposed technique was conducted to investigate the effect of fuzzy adaptation in Web applications based on the cognitive characteristics of users.

It was demonstrated that users' information finding ability was slightly more accurate and efficient in the adapted version rather than the original version of the same application. The observation was made in terms of both providing correct answers to the questions asked and in task completion time (performance). Additionally, users preferred the personalized version of the environment and results revealed that the majority of users could find the information they were seeking much 
easier and faster. These findings are encouraging and pave the path for further research and analysis since in general users' preference leans towards the personalized version indicating that the proposed mechanism has provided appropriate adaptation effects in relation to the cognitive characteristics of users.

Future research prospects include conducting further studies with a bigger sample in order to establish a more rigid connection between human factors and information processing in Web applications. In addition, further user studies with non computerexpert users are considered to analyze user performance and obtaining larger variability of the results.

Acknowledgements. The work is co-funded by the EU project Co-LIVING (6061700-98-009), smarTag (University of Cyprus) and PersonaWeb (Cyprus Research Promotion Foundation).

\section{References}

1. Brusilovsky, P.: Adaptive Hypermedia. J. User Modeling and User-Adapted Interaction 11(1,2), 87-110 (2001)

2. Germanakos, P., Tsianos, N., Lekkas, Z., Mourlas, C., Samaras, G.: Realizing Comprehensive User Profile as the Core Element of Adaptive and Personalized Communication Environments and Systems. The Computer Journal, SI on Profiling Expertise and Behaviour 52(7), 749-770 (2008)

3. Riding, R.: Cognitive Style Analysis - Research Administration. Learning and Training Technology (2001)

4. Smyth, B.: Case-based Recommendation. In: Brusilovsky, P., Kobsa, A., Nejdl, W. (eds.) Adaptive Web 2007. LNCS, vol. 4321, pp. 342-376. Springer, Heidelberg (2007)

5. Pazzani, M., Billsus, D.: Content-based Recommendation Systems. In: Brusilovsky, P., Kobsa, A., Nejdl, W. (eds.) Adaptive Web 2007. LNCS, vol. 4321, pp. 325-341. Springer, Heidelberg (2007)

6. Xiaoyuan, S., Khoshgoftaar, T.: A Survey of Collaborative Filtering Techniques. In: Advances in Artificial Intelligence, vol. 2009, ID 421425, 19 p. Hindawi Publishing Corp. (2009)

7. Zadeh, L.: Fuzzy Set. Information and Control 8(3), 338-353 (1965)

8. Felder, R., Silverman, L.: Learning and Teaching Styles in Engineering Education. Engineering Education 78, 674-681 (1988)

9. Witkin, H.A., Moore, C.A., Goodenough, D.R., Cox, P.W.: Field-dependent and fieldindependent cognitive styles and their educational implications. Review of Educational Research 47(1), 1-64 (1977)

10. Baddeley, A.D.: The concept of working memory: A view of its current state and probable future development. Cognition 10(1-3), 17-23 (1981)

11. Riding, R.J., Cheema, I.: Cognitive styles - An Overview and Integration. J. Educational Psychology 11(3/4), 193-215 (1991)

12. Baddeley, A.D.: Working Memory. Science 255, 556-559 (1992)

13. Bishop, C.: Neural Networks for Pattern Recognition. Clarendon, Oxford (1995)

14. Yager, R., Zadeh, L.: Fuzzy Sets, Neural Networks, and Soft Computing. Van Nostrand Reinhold, New York (1994)

15. Kirakowski, J., Cierlik, B.: Measuring the Usability of Web sites. In: Proceedings of Human Factors and Ergonomics Society 42nd Annual Meeting, Santa Monica, CA (1998) 